\title{
The effect of ischaemic region shape on epicardial potential distributions in transient models of cardiac tissue
}

\author{
J. P. Barnes ${ }^{1} \quad$ P. R. Johnston ${ }^{2}$
}

(Received 9 January 2012; revised 1 May 2012)

\begin{abstract}
Cardiac ischaemia is a restriction of blood supply to tissues in the heart. It is often diagnosed via an electrocardiograph by looking at the segment of the electrocardiograph between the QRS complex and the T wave, which is known as the ST segment. Previous work on ischaemia during the ST segment has shown that the shape of the ischaemic region can significantly affect the epicardial potential distribution at the surface of the heart. This numerical study compares the results from the steady state simulations with a more realistic transient model. The model consists of a slab of ventricular tissue with an ischaemic region at the centre resting on a blood bath. The transient bidomain equations were solved using a finite volume method for the spatial discretisation and a semi-implicit method for the time
\end{abstract}

http://journal . austms.org.au/ojs/index.php/ANZIAMJ/article/view/5101 gives this article, (c) Austral. Mathematical Soc. 2012. Published May 9, 2012. ISSN 1446-8735. (Print two pages per sheet of paper.) Copies of this article must not be made otherwise available on the internet; instead link directly to this URL for this article. 
integration. Ischaemia is included by taking into account three of the main physiological consequences which include hyperkalaemia, acidosis and anoxia. Results are obtained for three different ischaemic region geometries (rectangular, cylindrical and semi-ellipsoidal).

\section{Contents}

1 Introduction

2 Methods

2.1 Slab model . . . . . . . . . . . . . . . . . .

2.2 Governing equations . . . . . . . . . . . . . .

2.3 Cell model . . . . . . . . . . . . . . . . . . . .

2.4 Solution procedure . . . . . . . . . . . .

3 Results

4 Conclusion

References
C111

C113

$\mathrm{C} 113$

C114

C116

C121

$\mathrm{C} 122$

C124

\section{Introduction}

Depression of the ST segment in the electrocardiogram has long been recognised as an indicator of subendocardial ischaemia [18]. However, the mechanisms responsible for this behaviour are still not fully understood [11]. A previous steady state study [1] has shown that ST potentials are significantly affected by the geometry of the ischaemic region. This article compares the results from the steady state study to a more realistic transient model.

Numerical computational models of cardiac tissue have gained in popularity in recent years due to the advancement of computing technology $[5,4,7,10]$. 
This is due to numerical models being able to examine complex scenarios quickly and non-invasively, unlike their experimental counterparts. These numerical studies have consistently shown that for the case where ischaemia extends all the way to the epicardium (full thickness), elevation of electric potential occurs over the ischaemic region. For the case where the ischaemia has not reached the epicardium, the results start to vary. This is partially due to the lack of experimental data for vital parameters, such as conductivity. As cardiac tissue is anisotropic in nature, it is important to accurately measure the conductivity for each of the major axes directions.

This study is interested in the electrical consequences of ischaemia during the ST segment of the electrocardiograph (ECG). This is the phase of the cardiac cycle in which the normal heart is completely depolarised and is in an isoelectric state. The presence of an ischaemic region in the heart affects the electrical properties of cardiac cells and causes the heart to no longer be isoelectric during this phase. This effect can be measured by the extracellular potentials at the heart surface or as a deflection of the ST segment on an ECG trace. The ischaemic region itself is usually caused by a blockage or narrowing of one of the arteries which supplies blood to the heart tissue. This causes a lack of oxygen and nutrients in the cells and also a build up of waste, which affects the ionic processes that govern the cells electrical properties.

One consistent aspect of numerical studies of ST segment deviation is the use of a 'rectangular' shape to represent the ischaemic geometry. A previous study into the shape of ischaemic regions [1] showed that ischaemic geometry is important when modelling ischaemia during the ST segment. This study used a steady state bidomain model to compare the extracellular epicardial potential distributions for three different ischaemic geometries. For the rectangular and cylindrical geometry, it was found that a single depression occurs over the ischaemic region when the thickness of the ischaemic region is less than $30 \%$. As the thickness is increased, the single depression separates into three depressions, one over the ischaemic region and two over opposing lateral borders. Once the ischaemic thickness reaches $70-75 \%$, elevation appears on the inside of the lateral borders. This elevation then quickly 
spreads to cover the area over the ischaemic region as the thickness increases to full thickness.

The ellipsoidal ischaemic geometry displays similar qualitative behaviour for low thicknesses. However, the results start to differ once the ischaemic thickness increases above 30\%. Instead of the single depression separating into three depressions, only two depressions occur with the absence of the central depression. As the thickness increases beyond 70\%, elevation occurs again over the the ischaemic region.

For the previous steady state study [1], the ischaemic region was constructed by forcing the transmembrane potential to be lower inside the ischaemic region than outside. The motivation for the current study is to consider a more realistic transient situation and explore whether the epicardial potential distributions are still affected by the ischaemic geometry in the same way. This means that the transmembrane potentials need to be calculated using a complex model which considers the different ionic processes occurring within a cardiac cell. Ischaemia could then be induced by altering the cell model to match experimental results.

\section{Methods}

\section{$2.1 \quad$ Slab model}

The numerical model used in this study is based on the slab model introduced by Johnston et al. [5]. It describes a slab of ventricular tissue $1 \mathrm{~cm}$ thick with infinite length and width. This slab then sits on a mass of blood of infinite length. As this study is numerical in nature, the dimensions of the slab needed to be finite, so a length of $16 \mathrm{~cm}$ in the $x$ and $y$ directions were used and the blood thickness was set to $20 \mathrm{~cm}$. In the original slab model, the ischaemic region was represented by a rectangular prism with a length and width of $2 \mathrm{~cm}$ and was situated at the centre of the endocardium. For 
this study, two additional ischaemic geometries were considered. The first was represented by a cylinder with a radius of $2 \mathrm{~cm}$ and the second by the top hemisphere of an oblate spheroid with a semi-major axis of $2 \mathrm{~cm}$. Figure 1 shows a diagram of the slab model with the different ischaemic geometries.

\subsection{Governing equations}

The bidomain equations [17] are widely used with studies involving cardiac electrical activity. One representation of the bidomain equations has the following form. In the cardiac tissue $(0 \leqslant z \leqslant 1)$, the equations governing the electric potential are

$$
\begin{aligned}
\nabla \cdot\left(M_{i}+M_{e}\right) \nabla \phi_{e} & =-\nabla \cdot M_{i} \nabla \phi_{m} \\
\nabla \cdot M_{i} \nabla \phi_{m}+\nabla \cdot M_{i} \nabla \phi_{e} & =\beta\left[C_{m} \frac{\partial \phi_{m}}{\partial t}+i_{\text {ion }}\right] .
\end{aligned}
$$

Equation (1) is known as the steady state bidomain equation and Equation (2) describes the propagation of the transmembrane potential through the tissue [13]. Since the blood $(z>1)$ is a source free region, the electric potential there is governed by Laplace's equation,

$$
\nabla^{2} \phi_{\mathrm{b}}=0,
$$

where $\phi_{m}$ and $\phi_{e}$ are transmembrane and extracellular potentials, $M_{i}$ and $M_{e}$ are the intracellular and extracellular conductivities of the tissue, $\phi_{\mathrm{b}}$ is the blood potential, $C_{m}$ is the cell membrane capacitance and $\beta$ is the cell surface area to volume ratio. The $i_{\text {ion }}$ term represents a collection of ionic processes which occur within the cell and across the cell membrane. This term can be altered to mimic the behaviour of different types of cells depending on which part of the heart is of interest. The conductivity values used in this study were based on values given by Clerc [2]: $\sigma_{e}^{\mathrm{l}}=6.25 \mathrm{mS} / \mathrm{cm}, \sigma_{e}^{\mathrm{t}}=2.36 \mathrm{mS} / \mathrm{cm}$, $\sigma_{e}^{s}=2.36 \mathrm{mS} / \mathrm{cm}, \sigma_{i}^{l}=1.74 \mathrm{mS} / \mathrm{cm}, \sigma_{i}^{\mathrm{t}}=0.193 \mathrm{mS} / \mathrm{cm}, \sigma_{i}^{s}=0.193 \mathrm{mS} / \mathrm{cm}$. Linear fibre rotation was also included with a total rotation of $120^{\circ}$ from 


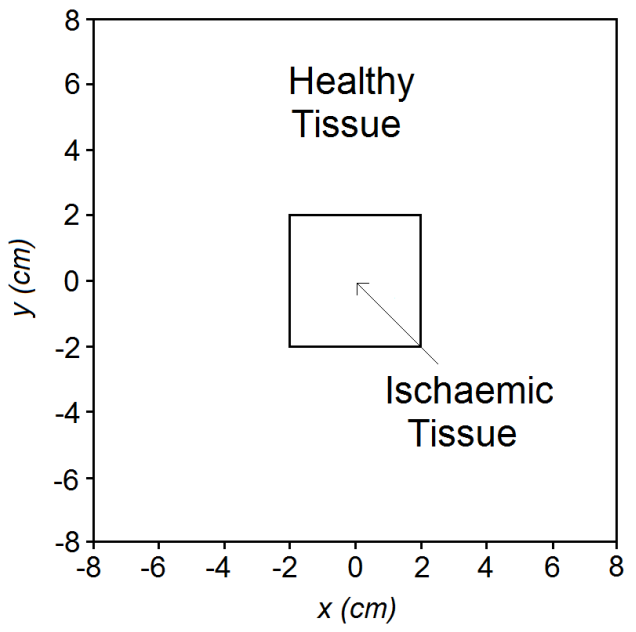

(a)

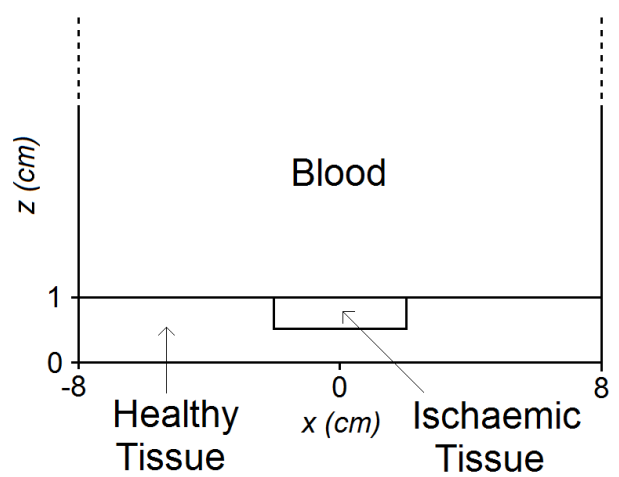

(c)

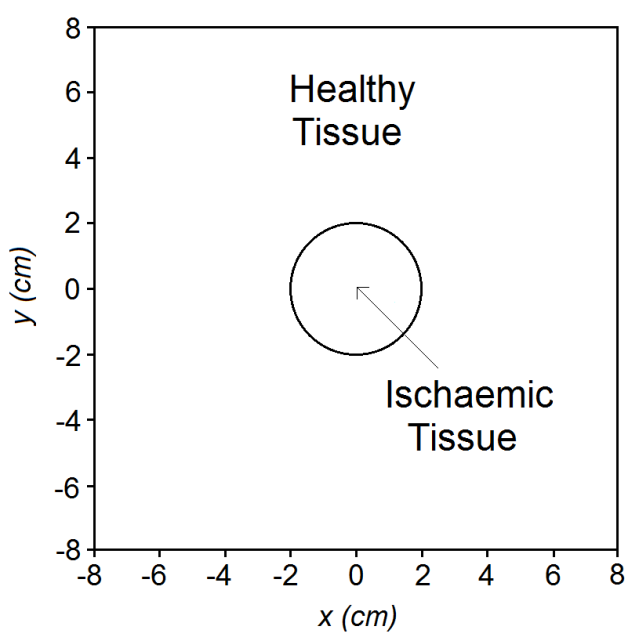

(a)

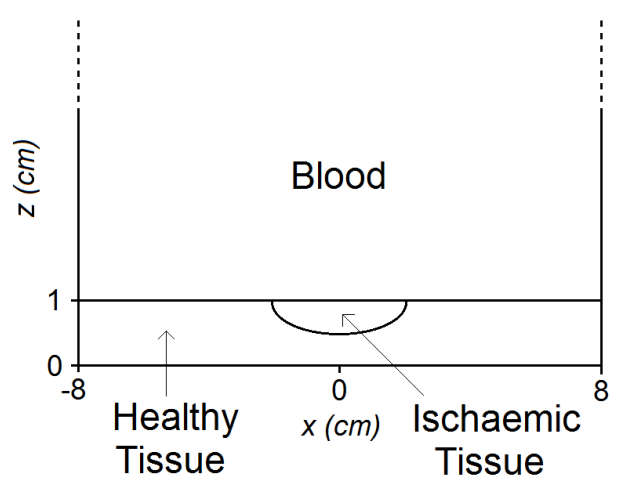

(d)

Figure 1: Slab model used for solving the bidomain equations. (a) cross section along $z$ axis for rectangular ischaemic geometry. (b) cross section along $z$ axis for cylindrical and ellipsoidal ischaemic geometries. (c) cross section along $y$ axis for rectangular and cylindrical ischaemic geometries. (d) cross section along $y$ axis for ellipsoidal ischaemic geometry. 
the endocardium to the epicardium (anti-clockwise direction). This was incorporated into the model by modifying the conductivity tensors to

$$
M_{n}=\left[\begin{array}{ccc}
\left(\sigma_{n}^{l}-\sigma_{n}^{t}\right) c^{2}+\sigma_{n}^{t} & \left(\sigma_{n}^{l}-\sigma_{n}^{t}\right) c s & 0 \\
\left(\sigma_{n}^{l}-\sigma_{n}^{t}\right) c s & \left(\sigma_{n}^{l}-\sigma_{n}^{t}\right) s^{2}+\sigma_{n}^{t} & 0 \\
0 & 0 & \sigma_{n}^{s}
\end{array}\right]
$$

where

$$
c=\cos \left(\frac{2 \pi}{3} z\right), \quad s=\sin \left(\frac{2 \pi}{3} z\right),
$$

and $n=i$ or $e$ for the intracellular or extracellular space. Conductivities $\sigma^{l}$, $\sigma^{t}$ and $\sigma^{s}$ represent the conductivity along the fibres, across the fibres and through the sheets respectively. Equations (1) and (3) form the steady state bidomain equations and can be used to find the extracellular potentials if the transmembrane potential distribution is known in advance. Equation (2) is an advection-diffusion equation which describes how the transmembrane potential distributions is affected by the ionic processes occurring at the cellular level.

At the blood-air and tissue-air interface (that is, the outer surface of the model), insulation conditions were assumed and at the tissue-blood interface, continuity of extracellular current and potential were assumed, giving the set of boundary conditions

$$
\begin{array}{ll}
\hat{\mathbf{n}} \cdot \nabla \phi=0, & \text { at the outer surface; } \\
\phi_{e}=\phi_{\mathrm{b}}, \quad \sigma_{e}^{\mathrm{t}} \frac{\partial \phi_{e}}{\partial z}=\sigma_{\mathrm{b}} \frac{\partial \phi_{\mathrm{b}}}{\partial z}, & \text { at the tissue-blood interface. }
\end{array}
$$

where $\hat{\mathbf{n}}$ is the unit vector perpendicular to the outer surface of the model.

\subsection{Cell model}

The electrical activity in the heart is mainly governed by the flow of ions between cells and across cell membranes. It is therefore important to carefully 
consider the effect of each ionic process on the transmembrane potential. In cardiac cells, certain ions may move across the cell membrane through complex protein structures known as channels (which permit a single ion species to passively diffuse through the membrane), exchangers (which are able to move one ion species into the cell whist removing another) and pumps (which actively move ions across the cell membrane using energy from intracellular adenosine triphosphate (ATP)). The net result of these transporters is an imbalance of charge between the intracellular and extracellular spaces which results in the transmembrane potential. These structures are not always active and may close to stop the flow of ions. This usually occurs when the transmembrane potential reaches some threshold but can also be affected by the concentration of certain ions within the cell. A computation cell model attempts to describe these ionic processes as currents across the cell membrane. Most of these currents are described in terms of ODEs which attempt to reproduce experimental results. The sum of all these processes makes up the $\mathfrak{i}_{\text {ion }}$ term in Equation (3). For this study, a cell model based on that by ten Tusscher et al. [16] was used, which considers the effect of twelve ionic currents and is decomposed as

$\mathfrak{i}_{\text {ion }}=\mathfrak{i}_{\mathrm{Na}}+\mathfrak{i}_{\mathrm{K} 1}+\mathfrak{i}_{\mathrm{to}}+\mathfrak{i}_{\mathrm{Kr}}+\mathfrak{i}_{\mathrm{Ks}}+\mathfrak{i}_{\mathrm{Ca} \mathrm{L}}+\mathfrak{i}_{\mathrm{Na} \mathrm{K}}+\mathfrak{i}_{\mathrm{b}_{\mathrm{Na}}}+\mathfrak{i}_{\mathrm{NaCa}}+\mathfrak{i}_{\mathrm{b}_{\mathrm{Ca}}}+\mathfrak{i}_{\mathrm{p}_{\mathrm{K}}}+\mathfrak{i}_{\mathrm{p}_{\mathrm{Ca}}}+\mathfrak{i}_{\mathrm{Stim}}$,

where

$i_{\mathrm{Na}}=$ fast sodium channel,

$\mathfrak{i}_{\mathrm{K} 1}=$ inward rectifier potassium channel,

$i_{\text {to }}=$ transient outward channel,

$\mathfrak{i}_{\mathrm{Kr}}=$ rapid time dependent potassium channel,

$i_{\mathrm{K} s}=$ slow time dependent potassium channel,

$\mathfrak{i}_{\mathrm{CaL}}=\mathrm{L}$ type calcium channel,

$i_{\mathrm{NaK}}=$ sodium potassium pump,

$i_{b_{\mathrm{Na}}}=$ sodium background channel,

$\mathfrak{i}_{\mathrm{NaCa}}=$ sodium calcium exchanger, 


$$
\begin{aligned}
\mathfrak{i}_{\mathrm{b}_{\mathrm{Ca}}} & =\text { calcium background channel, } \\
i_{\mathrm{p}_{\mathrm{K}}} & =\text { potassium pump } \\
\mathfrak{i}_{\mathrm{p}_{\mathrm{Ca}}} & =\text { calcium pump }
\end{aligned}
$$

The $i_{\text {Stim }}$ is usually added to a cell model to simulate external stimulation of a cell. For cells which are not being stimulated, this term is set to zero. Ten Tusscher et al. $[16,15]$ detail an explanation of each ionic process and the equations associated with them. In order to more accurately reproduce ischaemic conditions, an additional ATP activated $\mathrm{K}^{+}$current was added for this study using the formulation described by Shaw and Rudy [14]:

$$
\mathrm{I}_{\mathrm{K}, \mathrm{ATP}}=\mathrm{G}_{\mathrm{K}, \mathrm{ATP}} \frac{1}{1+\left([\mathrm{ATP}]_{\mathrm{i}} / \mathrm{K}_{0.5}\right)^{2}}\left(\frac{\left[\mathrm{K}^{+}\right]_{\mathrm{o}}}{5.4}\right)^{0.24}\left(\phi_{\mathrm{m}}-\mathrm{E}_{\mathrm{k}}\right),
$$

where $G_{K, A T P}$ is the maximum conductance for the current, $[A T P]_{i}$ is the intracellular ATP concentration, $\left[\mathrm{K}^{+}\right]_{\mathrm{o}}$ is the extracellular potassium concentration, and $E_{k}$ is the Nerst equilibrium potential for potassium. The parameter $K_{0.5}$ is given a value of 0.042 for healthy cells and 0.25 for ischaemic cells [14].

Cardiac ischaemia occurs when tissue is deprived of oxygen and nutrients, usually caused by a blockage or narrowing of the arteries which supply blood to the cells. The effect of this at a cellular level is the closing of gap junctions, reducing the flow of ions between cells, and disrupting the gates which control the exchange of ions through the cell membrane. Another problem caused by the lack of fresh blood is the build up of cellular waste, which also affects ionic processes.

The resting potential for a healthy ventricular cell is approximately $-85 \mathrm{mV}$. Once activated the potential quickly rises to approximately $30 \mathrm{mV}$, then plateaus for $250-300 \mathrm{~ms}$, before falling back down to its resting potential. The overall effect of ischaemia on the action potential is a higher resting potential, lower plateau potential and a shorter plateau duration. The three major experimentally observed consequences of ischaemia which were modelled in this study were: 
1. Hyperkalaemia - Elevated extracellular $\mathrm{K}^{+}$concentration, caused by the inability to remove cellular waste and responsible for an increase in resting potential;

2. Acidosis-Affects the fast sodium and L type calcium currents by reducing the conductance of the respective ion channels, resulting in decreased upstroke velocity;

3. Anoxia-Activates the ATP dependent $\mathrm{K}^{+}$current, caused by a rise in the extracellular $\mathrm{pH}$ levels, resulting in decreased action potential duration.

Hyperkalaemia was achieved by raising $\left[\mathrm{K}^{+}\right]_{\mathrm{o}}$ from $5.4 \mathrm{mM}$ to $12 \mathrm{mM}$. Acidosis was achieved by reducing the conductivity of the fast sodium channel by $25 \%$ and the conductivity of the L type calcium channel by 50\%. Anoxia was simulated by an increase of the $\mathrm{K}_{0.5}$ parameter as mentioned above. Figure 2 shows the transmembrane potential for a single cell using both healthy and ischaemic parameters. This plot was obtained by numerically solving the equation

$$
\mathrm{C}_{\mathrm{m}} \frac{\mathrm{d} \phi_{\mathrm{m}}}{\mathrm{dt}}=-i_{\text {ion }}
$$

with a stimulus applied at $t=10 \mathrm{~ms}$ for $2 \mathrm{~ms}$.

\subsection{Solution procedure}

The first step to solving the model was to spatially discretise the bidomain equations using a finite volume method. This was carried out as described by Johnston [6], which is based on a procedure by Penland [8]. The temporal solution consisted of a three step loop.

1. Using the transmembrane potentials from the $n$th time step $\left(\phi_{m}^{n}\right)$, the cell model equations were solved using a forward difference method to give the ionic current for the next time step $\left(i_{\text {ion }}^{n+1}\right)$. 


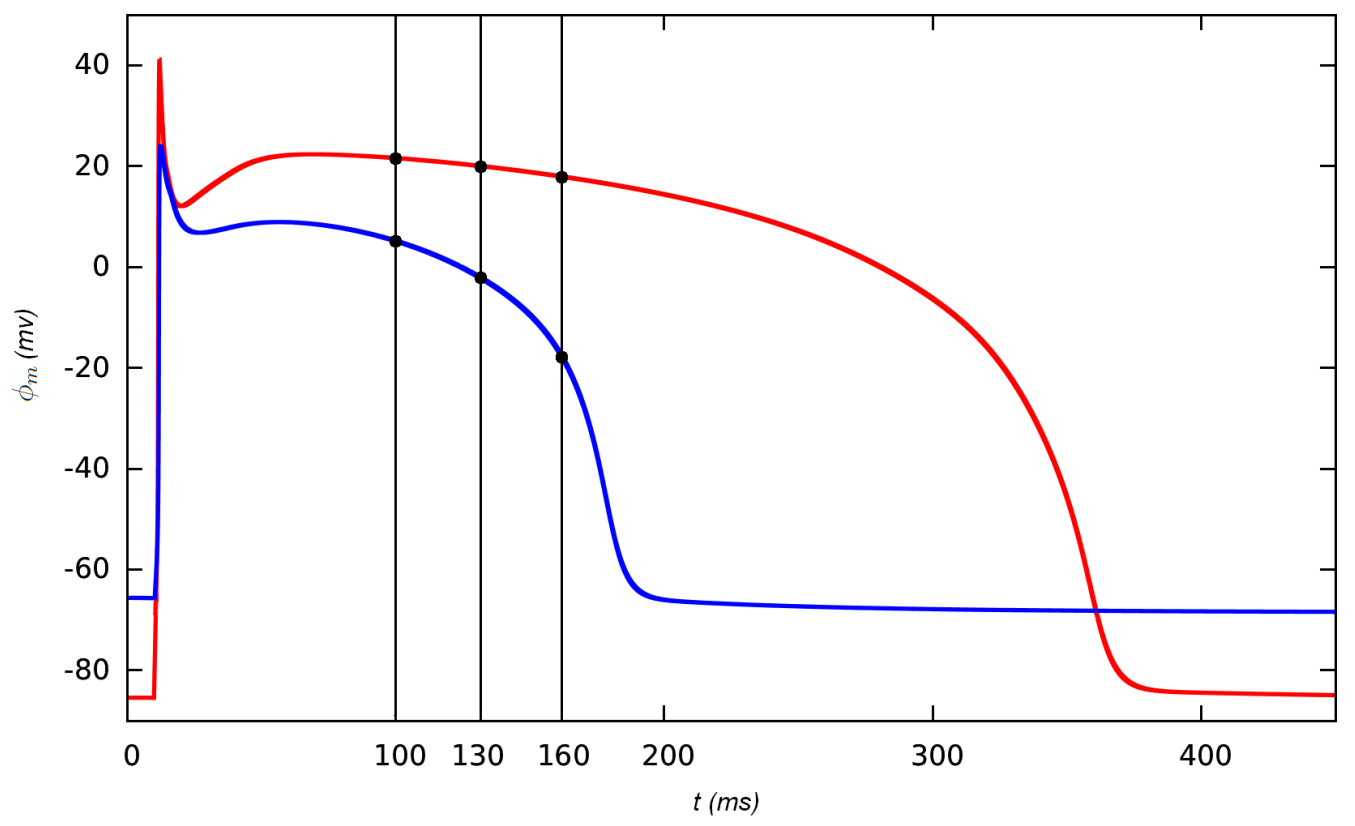

Figure 2: Action potential for healthy (red) and ischaemic (blue) tissue for a single cell. Vertical lines are added at $t=100, t=130$ and $t=160$ which correspond to the times used in Figure 3

2. Equations (1) and (3) were also solved using the transmembrane potentials from the $n$th time step to give the extracellular potentials for the next time step $\left(\phi_{e}^{\mathrm{n}+1}\right)$.

3. The extracellular potentials from step $2\left(\phi_{e}^{\mathrm{n}+1}\right)$ and ionic current from step $1\left(i_{\text {ion }}^{n+1}\right)$ were substituted into Equation (2) and the transmembrane potentials were updated using a backward difference method, giving $\left(\phi_{m}^{n+1}\right)$.

For the initial condition, all nodes were assumed to be in their resting state, so were given a transmembrane potential of $\phi_{\mathrm{m}}^{0}=-85 \mathrm{mV}$ for healthy nodes and $\phi_{\mathrm{m}}^{0}=-65 \mathrm{mV}$ for ischaemic nodes. All code was written in $\mathrm{C}++$ using the 
Petsc libraries in order to take advantage of the numerous parallel data types available. SCIRun [3] was also used to visualise the data at chosen time steps. The bidomain equations were solved using a time step of $0.1 \mathrm{~ms}$. However, the cell model needed a smaller time step to maintain numerical stability and so was updated every $0.01 \mathrm{~ms}$. In order to achieve an accurate solution, propagation simulations were run with an increasing number of nodes until the wave speed converged. We found that a node spacing of $0.5 \mathrm{~mm}$ was enough to ensure a reasonably accurate wave speed. A $\beta$ value of $1400 \mathrm{~cm}^{-1}$ [14] and a $C_{m}$ value of $1 \mu \mathrm{F}$ [9] were used for all simulations.

\section{$3 \quad$ Results}

Results were only obtained using an ischaemic thickness of $50 \%$. This was because the results from the steady state study showed that at low and high thicknesses, the ischaemic geometry had little effect on epicardial potential distributions [5]. Electrical propagation was achieved by stimulating the entire endocardium at $t=10 \mathrm{~ms}$ for $2 \mathrm{~ms}$. The endocardium was defined as the bottom $10 \%$ of tissue, where $0.9 \leqslant z \leqslant 1$. This propagation wave then travelled towards the epicardium at approximately $0.015 \mathrm{~cm} / \mathrm{ms}$. The time taken for the wave to travel completely through the tissue was approximately $60 \mathrm{~ms}$ for each of the three ischaemic geometries modelled. Figure 3 shows the epicardial potential distributions at three separate time points for each ischaemic geometry.

For the rectangular ischaemic geometry, once the entire tissue was depolarised, there was a slight depression apparent over the ischaemic region. At this point, the extra two depressions found in the steady state model were hardly noticeable. There was also slight elevation found over the left and right side of the ischaemic region. However, this behaviour did not remain for long. Once the transmembrane potentials began to stabilise at the epicardium, the elevation quickly disappeared and the two missing depressions began to form. At $t=130 \mathrm{~ms}$ the three depressions became quite distinct, and at $t=160 \mathrm{~ms}$ 
the results look qualitatively very similar to what was found in the steady state model.

The cylindrical model behaved quite similarly to its rectangular counterpart, which was expected. However, there was one noticeable difference between the two. The potentials at the central depression in the cylindrical model were higher than that of the rectangular model, which meant that the depression was not as distinct. This also differed from the steady state results, where the potentials in the central depression for both models were quite similar.

The ellipsoidal model showed some interesting behaviour early into the ST segment. From approximately $70-100 \mathrm{~ms}$, the results looked very similar to the two previous models, with a depression starting to form over the centre of the ischaemic region. The central depression then disappeared at approximately $110 \mathrm{~ms}$ along with the surrounding elevation and the epicardial potential distributions then started to form the familiar two depression pattern. The two depressions continued to deepen and once the time reached $160 \mathrm{~ms}$, the results were again very similar to the steady state model.

\section{Conclusion}

The results presented in this study clearly show that extracellular epicardial potential distributions are indeed affected by ischaemic geometry. Although there were similarities in the potentials for all models early in the ST segment, once the transmembrane potentials at the epicardium started to settle, the results quickly changed and began to look like the results from the previous steady state study. However, there were some elements which were not looked at in this study that could impact on the results. Further studies are needed to simulate the effect of conductivity changes during ischaemia, and also to look at more realistic heart models. Another simplification in this study was that the entire endocardium was depolarised simultaneously. A more realistic approach would be to simulate the addition of purkinje fibres, which carry 


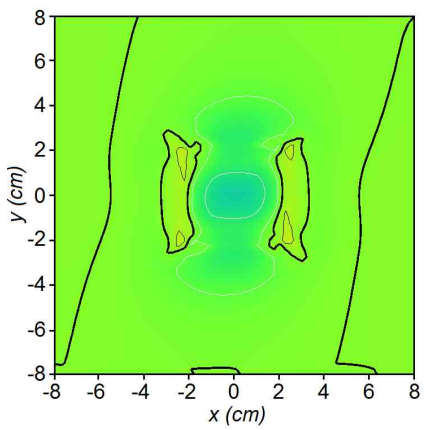

(a)

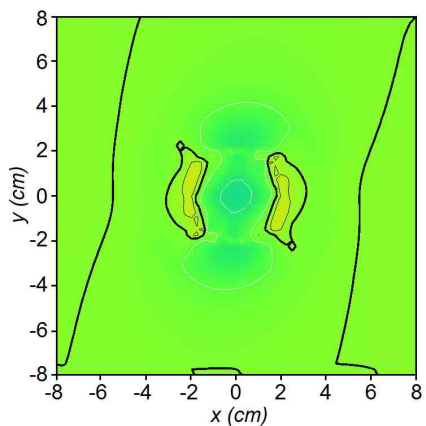

(d)

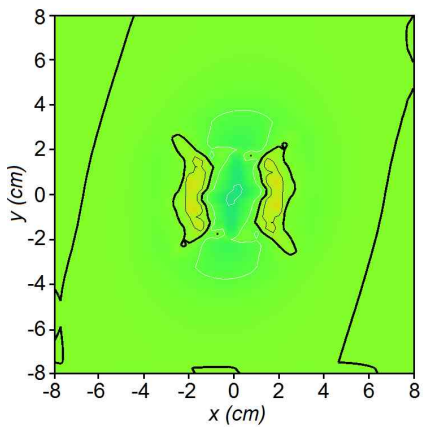

(g)

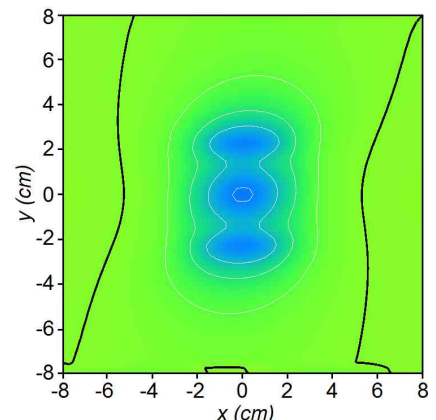

(b)

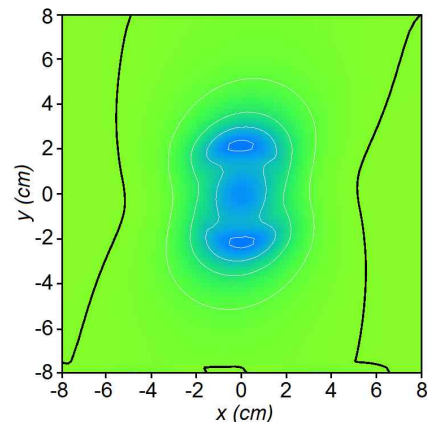

(e)

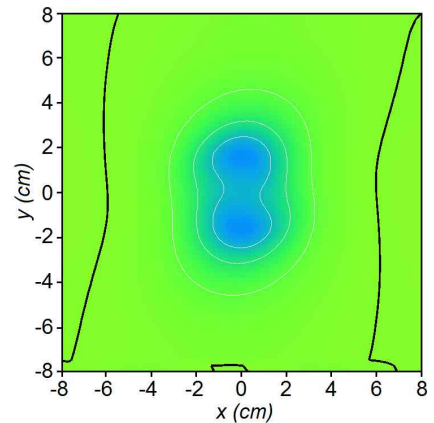

(h)

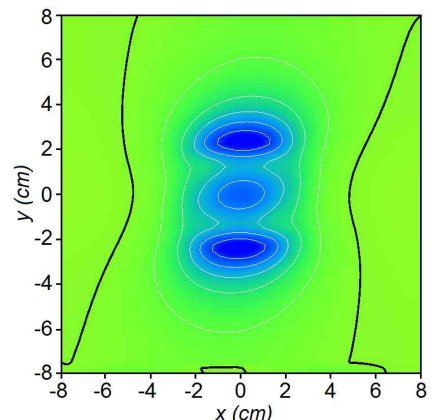

(c)

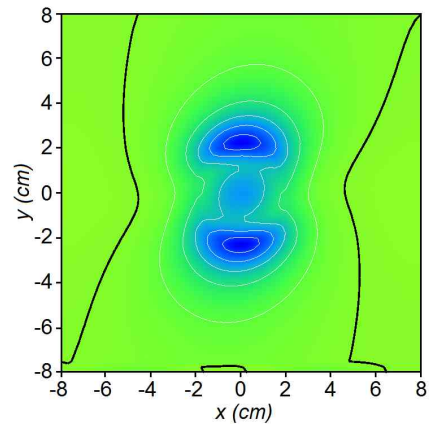

(f)

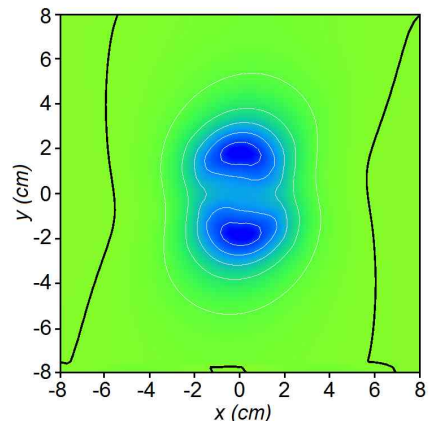

(i)

Figure 3: Extracellular epicardial potential distributions, $\phi_{e}(x, y, 0, t)$ for the rectangular (top row) cylindrical (middle row) and ellipsoidal (bottom row) ischaemic geometries. The columns from left to right correspond to $\mathrm{t}=100 \mathrm{~ms}, \mathrm{t}=130 \mathrm{~ms}$ and $\mathrm{t}=160 \mathrm{~ms}$. Black lines are positive potentials and white are negative potentials. The contour interval for all plots is $0.2 \mathrm{mV}$. 
the stimulus current throughout the endocardium [12].

Acknowledgements We gratefully acknowledge the support of the Griffith University eResearch Services Team and the use of the High Performance Computing Cluster Gowonda to complete this research.

\section{References}

[1] J. P. Barnes and P. R. Johnston. The effect of the shape of ischaemic regions in the heart on the resulting extracellular epicardial potential distributions. In Computing in Cardiology, 2010, pages 177-180, sept 2010. http://ieeexplore.ieee.org/xpl/articleDetails. jsp? arnumber $=5737938$. C111, C112, C113

[2] L Clerc. Directional differences of impulse spread in trabecular muscle from mammalian heart. The Journal of Physiology, 255(2):335-346, 1976. http://jp.physoc.org/content/255/2/335. C114

[3] Scirun: A scientific computing problem solving environment, Scientific Computing and Imaging Institute. http://www.scirun.org. C121

[4] P. R. Johnston and D. Kilpatrick. The effect of conductivity values on st segment shift in subendocardial ischaemia. Biomedical Engineering, IEEE Transactions on, 50(2):150-158, feb 2003. doi:10.1109/TBME.2002.807660 C111

[5] P. R. Johnston, D. Kilpatrick, and Chuan Yong Li. The importance of anisotropy in modeling st segment shift in subendocardial ischaemia. Biomedical Engineering, IEEE Transactions on, 48(12):1366-1376, dec 2001. doi:10.1109/10.966596 C111, C113, C121

[6] Peter R. Johnston. A finite volume method solution for the bidomain equations and their application to modelling cardiac ischaemia. 
Computer Methods in Biomechanics and Biomedical Engineering, 13(2):157-170, 2010. doi:10.1080/10255840903067072 C119

[7] Robert S. MacLeod, Shibaji Shome, Jeroen Stinstra, Bonnie B. Punske, and Bruce Hopenfeld. Mechanisms of ischemia-induced st-segment changes. Journal of Electrocardiology, 38(4, Supplement):8-13, 2005. doi:10.1016/j.jelectrocard.2005.06.095 C111

[8] R. Christian Penland, David M. Harrild, and Craig S. Henriquez. Modeling impulse propagation and extracellular potential distributions in anisotropic cardiac tissue using a finite volume element discretization. Computing and Visualization in Science, 4:215-226, 2002. doi:10.1007/s00791-002-0078-4 C119

[9] A. E. Pollard, N Hooke, and C. S. Henriquez. Cardiac propagation simulation. Crit Rev Biomed Eng, 20(3-4):171-210, 1992. http://ukpmc.ac.uk/abstract/MED/1478091. C121

[10] Mark Potse, Ruben Coronel, Stephanie Falcao, A.-Robert LeBlanc, and Alain Vinet. The effect of lesion size and tissue remodeling on st deviation in partial-thickness ischemia. Heart Rhythm, 4(2):200-206, 2007. doi:10.1016/j.hrthm.2006.10.022 C111

[11] Blanca Rodriguez, Natalia Trayanova, and Denis Noble. Modeling cardiac ischemia. Annals of the New York Academy of Sciences, 1080(1):395-414, 2006. doi:10.1196/annals.1380.029 C111

[12] Daniel Romero, Rafael Sebastian, Bart Bijnens, Viviana Zimmerman, Patrick Boyle, Edward Vigmond, and Alejandro Frangi. Effects of the purkinje system and cardiac geometry on biventricular pacing: A model study. Annals of Biomedical Engineering, 38:1388-1398, 2010. doi:10.1007/s10439-010-9926-4 C124

[13] B. J. Roth. Action potential propagation in a thick strand of cardiac muscle. Circulation Research, 68(1):162-173, 1991. doi:10.1161/01.RES.68.1.162 C114 
[14] Robin M. Shaw and Yoram Rudy. Electrophysiologic effects of acute myocardial ischemia: a theoretical study of altered cell excitability and action potential duration. Cardiovascular Research, 35(2):256-272, 1997. doi:10.1016/S0008-6363(97)00093-X C118, C121

[15] K. H. W. J. ten Tusscher, D. Noble, P. J. Noble, and A. V. Panfilov. A model for human ventricular tissue. American Journal of Physiology - Heart and Circulatory Physiology, 286(4):H1573-H1589, 2004. doi:10.1152/ajpheart.00794.2003 C118

[16] K. H. W. J. ten Tusscher and A. V. Panfilov. Alternans and spiral breakup in a human ventricular tissue model. American Journal of Physiology - Heart and Circulatory Physiology, 291(3):H1088-H1100, September 2006. doi:10.1152/ajpheart.00109.2006 C117, C118

[17] Leslie Tung. A bi-domain model for describing ischemic myocardial d-c potentials. PhD thesis, Massachusetts Institute of Technology, 1978. http://hdl.handle.net/1721.1/16177. C114

[18] Charles C. Wolferth, Samuel Bellet, Mary M. Livezey, and Franklin D. Murphy. Negative displacement of the rs-t segment in the electrocardiogram and its relationships to positive displacement; an experimental study. American Heart Journal, 29(2):220-245, 1945. doi:10.1016/0002-8703(45)90519-9 C111

\section{Author addresses}

1. J. P. Barnes, School of Biomolecular and Physical Sciences, Griffith University, Queensland 4111, Australia. mailto: josef . barnes@griffithuni.edu.au

2. P. R. Johnston, School of Biomolecular and Physical Sciences, Griffith University, Queensland 4111, Australia. 